\title{
Quality of life measurement and outcome in aphasia
}

This article was published in the following Dove Press journal:

Neuropsychiatric Disease and Treatment

16 December 2013

Number of times this article has been viewed

\section{Simona Spaccavento \\ Angela Craca \\ Marina Del Prete \\ Rosanna Falcone \\ Antonia Colucci \\ Angela Di Palma \\ Anna Loverre}

IRCCS Salvatore Maugeri

Foundation, Cassano Murge Bari, Italy
Background: Quality of life (QL) can be defined as the individual's perception of their own well-being. Aphasia is the most important potential consequence of stroke and has a profound effect on a patient's life, causing emotional distress, depression, and social isolation, due to loss of language functions.

Aims: To draw up a QL questionnaire for aphasics (QLQA) focusing particularly on difficulties in interpersonal relationships and on the loss of independence as a result of language disorders. We reported the results of a psychometric evaluation of this measure. Moreover, we experimentally focused on the differences in QLQA between patients affected only by neurological motor impairment and hemiparetic patients with aphasia (PWA) in order to verify the specific role of aphasia on QL. We also explored if the QLQA is sensitive to the severity of aphasia and to the time elapsing from the stroke.

Methods: A total of 146 consecutive PWA and 37 control subjects were enrolled to evaluate the reliability (internal consistency and test-retest reliability) and validity of the QLQA, using standard psychometric methods. Patients were divided into acute (within 3 months since stroke) and chronic (beyond 3 months) groups, and into mild and severe according to the severity of aphasia. The experimental group of only acute PWA was compared to control subjects, with right hemispherical lesion and without aphasia in QLQA total and partial scores.

Results: The QLQA had good internal consistency and test-retest reliability. Acute and chronic PWA and mild and severe ones differed in QLQA total, communication, and autonomy subscales. No differences were found in psychological condition. Between aphasic and control patients, significant differences were found in all QLQA subscales.

Conclusion: The QLQA is a valid measure of QL in PWA, contributing to a better distinction between severe and mild aphasia, and it is sensitive also to the variations in QL depending on the time interval from stroke.

Keywords: aphasia, quality of life, outcome, rehabilitation

\section{Introduction}

Quality of life (QL) may be defined as an individual's perception of their well-being. Health-related QL reflects the impact of a healthy state on a person's ability to lead a fulfilling life, and covers the individual's satisfaction in physical, functional, psychological, and social domains. ${ }^{1}$

Studies ${ }^{2,3}$ have shown that QL worsens after brain damage (vascular lesions, head injury, infections). Changes in QL in the poststroke period do not seem to be age- ${ }^{4}$ or sex-dependent, but rather are due to the onset of depression ${ }^{5}$ and a low level of reacquisition in motor functions, above all in the upper limbs. ${ }^{6}$ QL improves within the family environment.?
Correspondence: Simona Spaccavento IRCCS Salvatore Maugeri Foundation, Via per Mercadante Km 2, 70020 Cassano Murge, Bari, Italy

$\mathrm{Tel}+3908078$ I 426।

$\mathrm{Fax}+390807814310$

Email simona.spaccavento@fsm.it 
Aphasia is the loss of or radical change in voluntary speech as a consequence of damage to the left cerebral hemisphere, and generally causes significant changes in a patient's self-image and family, social, and work relationships. ${ }^{8}$ The impairment in language skills due to aphasia causes problems in verbal expression, auditory comprehension, reading, and writing. Aphasia is a prominent cause of limitation on communication activities, such as using the phone or writing a letter. Aphasia will have relatively little direct impact upon the performance of domestic activities of daily living, but it will particularly affect complex social activities, such as work and participating in community activities and leisure activities involving other people. Studies have documented high levels of depression ${ }^{9}$ and social exclusion, ${ }^{10}$ and low levels of leisure and other social activities, ${ }^{11}$ social contacts, ${ }^{12}$ and $\mathrm{QL}^{13,14}$ among patients with aphasia (PWA). Significant correlations have been found between degree of aphasia and the social, emotional, mobility, and total scores of QL, measured with the Nottingham Health Profile. ${ }^{15}$

In a population-based study, Lam and Wodchis ${ }^{16}$ found that aphasia exhibited the largest negative influence on QL, followed by cancer and Alzheimer's disease. PWA reported significantly worse QL than nonaphasic patients, since aphasia influenced independence, social relationships, and access to their environment.

Le Dorze and Brassard ${ }^{17}$ have described the consequences of aphasia by analyzing the personal accounts of aphasic individuals, relatives, or friends. These consequences were classified into one of three categories: disabilities, handicaps, and coping behaviors. In the first group, language disabilities and aphasia-linked difficulties were included. The handicaps included changes in situations of communication, in interpersonal relationships, loss of autonomy, and restriction of activities. Coping behaviors, however, were adopted by PWA and their relatives to adjust the undesired effects of the various disabilities and handicaps they experienced.

Although a number of stroke-specific QL scales have been developed, most exclude stroke survivors with aphasia, subjects most prone to social isolation and exclusion. This is due to the difficulties faced in proposing a questionnaire to subjects with severe comprehension and expression deficits. In regard to the studies on the impact of aphasia on patient's life, there are two paradigms. From a qualitative perspective, ethnographic methods, like participant and nonparticipant observations and analysis of artifacts such as diaries, can reflect the everyday experiences of PWA and their social inclusion, but they do not suggest how patients feel their
QL to be. From a quantitative perspective, many authors ${ }^{18,19}$ have evaluated the QL of patients with severe aphasia, asking proxy respondents to report on their partners' health-related quality of life (HRQL). Also in this case, evaluating one's QL is highly subjective.

In a recent meta-analysis, ${ }^{1}$ only 14 research studies reported factors associated with QL in PWA. Emotional distress, aphasia severity, communication and activity limitations, other medical problems, and social aspects all influenced QL. The extent of aphasia (severity, language impairment, communication disability) was associated with or predictive of lower HRQL in seven of the eight reviewed studies.

Hilari et a ${ }^{20}$ evaluated the QL in patients with and without aphasia, testing a stroke-specific HRQL scale (the Stroke and Aphasia Quality of Life Scale [SAQOL-39]) in a generic stroke sample that included patients both with and without aphasia. The SAQOL-39 generic stroke scale measures HRQL after stroke in three domains: physical, psychosocial, and communication. This scale demonstrates good internal consistency, test-retest reliability and validity, and adequate responsiveness to changes in a sample of patients with acute and chronic stroke.

The information obtained from QL measures can be useful to identify patients' problems, determine treatment priorities, manage interventions, and monitor disease periods.

The first aim of this observational study was to conduct a preliminary evaluation of the psychometric properties (validity, reliability) of a QL questionnaire for aphasics (QLQA), in which we focused particularly on difficulties in interpersonal relationships, on loss of independence, and on abilities in daily life as a result of language disorders. Moreover, in the aphasic group, we studied how QLQA was sensitive to the severity and type of aphasia and to the time from onset. Finally, we tried to identify the specific role of linguistic deficits on QL, minimizing the effects of motor impairment. The QLQA scores of PWA with hemiparesis were compared to those of a control group affected by a right-brain injury or a neurological peripheral disease and motor impairment, but with no linguistic or communicative problems.

\section{Materials and methods Subjects}

A total of 164 consecutive patients with neurological central disease admitted to our Neurorehabilitation Unit for cognitive and physical rehabilitation from 2011 to 2012 were examined. Eighteen of them were excluded for bilateral 
or multiple lesions identified by neurodiagnostic scans (computerized axial tomography or magnetic resonance imaging). The experimental group consisted of 146 PWA and right hemiparesis, all cared for by a caregiver. They had one left-hemisphere cerebral lesion confirmed by neurodiagnostic studies. Inclusion criteria were aphasia resulting from unilateral left-hemisphere stroke, unknown prestroke history of severe cognitive decline, or mental health problems. No patients presented with prestroke neuropsychological deficits, psychiatric disorders, history of alcohol or drug abuse, head injury, or tumoral lesions. Patients were excluded if they did not speak Italian premorbidly. A control group consisted of 37 subjects with right-hemisphere cerebral damage, hemiparesis, and varying degrees of unilateral spatial neglect.

Written consent was obtained from all patients. The study was approved by the internal Salvatore Maugeri Foundation's ethics committee. The study was conducted in accordance with the principles of the Declaration of Helsinki. All the patients underwent an initial screening to evaluate the presence and type of linguistic disorders and the severity of motor impairment.

\section{Materials}

All aphasic patients were evaluated by the Aachener Aphasia Test (AAT) ${ }^{21}$ to analyze language deficits and to diagnose type of aphasia. For this study, the patients underwent the following series of tests, administered by a speech therapist:

- the subtest of spontaneous speech of the AAT, ${ }^{21}$ structured in six parts (communicative behavior, articulation and prosody, automatic language, semantics, phonology, syntax), with a score range from 0 to 5

- the Token Test ${ }^{22}$ to assess verbal comprehension with 36 items

- Raven's Coloured Progressive Matrices, ${ }^{23,24}$ which provides a nonverbal measure of intellectual abilities; the patient must logically complete a given visual spatial pattern, choosing from a set of six alternatives

- a scale of functional independence - the Functional Independence Measure ${ }^{25,26}$ (FIM) - divided into scores for motor FIM (13 items) and cognitive FIM (five items)

- another scale of functional independence - the Functional Assessment Measure (FAM) ${ }^{27-29}$ cognitive subscale a compound of fourteen items evaluating comprehension, expression, reading, writing, speech intelligibility, social interaction, emotional status, adjustment to limitation, employability, problem solving, memory, orientation, attention span, and safety judgment

- the QLQA.

\section{Quality of life questionnaire for aphasics}

HRQL questionnaires give outcome measures that evaluate the impact of health on a person's ability to lead a fulfilling life, and generally incorporate the individual's perceptions of physical, mental/emotional, family, and social functioning. Although a number of stroke-specific QL scales have been developed, measures that ecologically identify both the effects of linguistic communicative disability and the quality of everyday life are still needed for PWA. They could be useful to bridge the gap between linguistic rehabilitation and the patient's real use of residual means of communication or ability to perform the daily life activities in which language is involved. In this way, they could link the individual rehabilitation training to outcomes of increased functional autonomy in social and communicative environments, according to the recent World Health Organization International Classification of Functioning, Disability and Health (ICF). ${ }^{30}$

One example of these measures is the Functional Outcome Questionnaire for Aphasia, ${ }^{31}$ used in the US. It consists of 32 items, and is focused on the efficacy of the aphasic's verbal and nonverbal communicative abilities. This scale assesses the ability to communicate basic needs and new information and the ability to make routine requests; it also investigates the issues of communication and comprehension. The SAQOL-39 ${ }^{32}$ scale, recently translated into Italian and also into Spanish, ${ }^{33,34}$ is a more complete QL assessment (it includes measurement of general, physical, and psychosocial health and vitality), even if there are few linguistic items compared to the total number (nine of 39). Our QLQA has the aim of addressing a lack of QL measures in the Italian language, including, as with previous ones, measurement of several domains, such as physical, psychological, communication, and social participation.

Engell et $\mathrm{al}^{35}$ developed a pictorial procedure for rating QL to minimize the influence of aphasia. They transformed an existing QL inventory - the modified German version of the Sickness Impact Profile ${ }^{36}$ - into a picture-based representation. The authors selected the Aachen Quality of Life Inventory (ALQI), ${ }^{37}$ a German-language adaptation of the Sickness Impact Profile. The items assessed psychosocial and physical dimensions, language, and cognition. The ALQI items were transformed into a pictorial version to maximize aphasic patients' understanding of the verbal statements, as well as to permit them to give nonverbal responses. All pictures are professionally rendered simple line-drawings. Additional pictograms permitted the patients to indicate their ratings. This procedure allowed for self-rating of aphasic patients in parallel to a proxy rating by caregivers. There was 
in fact also a written version for the caregivers that corresponded item by item to the patients' pictorial version.

The limited use of these assessments has negative effects on the course of clinical welfare. In Italy, stroke rehabilitation still focuses more on the improvement of physical autonomy rather than on a global increase in neuropsychological functions, which are often affected. Rehabilitation therapy should aim not only to regain communicative linguistic abilities, but also to help in adapting to the disability and to encourage social integration and personal well-being, thus improving QL.

Our QL questionnaire was developed by means of the aforementioned conceptual considerations and analysis of literature studies and of the previous QL and functional questionnaires for aphasic patients, in order to point out the aphasic disease, handicap and coping behavior in everyday life. The questionnaire consists of 37 questions that are important for daily well-being, chosen from a larger repertoire by a mixed group of PWA and their caregivers and members of the Italian Aphasia Association of our region (Puglia). We consequently based the QLQA more on the percentage of residual abilities performed by the individuals with aphasia rather than on their perceived $\mathrm{QL}$, which was difficult to investigate, considering their communicative problems.

The QLQA is able to:

- express the disability, mainly as relationship problems and loss of autonomy caused by linguistic deficits; it consists in a majority of questions regarding languagerelated activities and abilities

- consider verbal comprehension difficulties

- include items of residual functional disability due to motor deficits

- include items on psychological problems that occur both in the poststroke phase and later, partly as a result of brain damage but also the social and individual aftereffects of aphasia

- highlight relational and nonrelational residual problems due to language deficits.

The questions, formulated as easily and succinctly as possible, assess the ability to perform basic functions and convey health problems, the psychological changes due to the disability and the ability to socialize, the ability of linguistic and contextual comprehension, and expression in routine daily activities.

The items are scored on a 5-point scale, with $0=$ the individual is able to successfully perform the behavior $0 \%$ of the time, $1=$ the individual is able to successfully perform the behavior $25 \%$ of the time, $2=$ the individual is able to successfully perform the behavior $50 \%$ of the time, $3=$ the individual is able to successfully perform the behavior $75 \%$ of the time, and $4=$ the individual is able to successfully perform the behavior $100 \%$ of the time.

The QLQA was administered by a speech therapist using verbal or pragmatic means (gestures and drawings) when the patient showed comprehension deficits, in the presence of the caregiver. When there was no agreement in the answers, we considered those of the caregiver more reliable, so excluding the influence of the patients' reduced consciousness of their own limits or anxiety/depression state. The QLQA score was calculated by summing the items. High scores indicate better HRQL.

\section{Psychometric analysis}

Demographic and clinical variables of aphasic sample and control subjects were evaluated with descriptive statistics (means, standard deviations, etc), using Student's $t$-test for analyzing the differences between two samples. Due to the ordinal nature of the scale, differences in the QLQA scores and in other tests of both groups were analyzed using nonparametric tests (Mann-Whitney $U$ ). We used standard psychometric methods ${ }^{38-40}$ to evaluate internal consistency, test-retest reliability, and construct validity (convergent and discriminant) of QLQA in 146 aphasic patients.

A principal-component analysis (PCA, a type of factor analysis) was used to determine the number and the type of domains underlying the QL of aphasic patients and reduce the number of items in the QLQA to those best measuring it.

In order to evaluate convergent and discriminant validity, a multiple correlation among the scores of the QLQA subtests (communication, autonomy, and psychological condition), resulting from PCA, and the FIM and FAM scores and the correct linguistic tests scores were calculated by Spearman's correlation coefficient. Internal consistency was calculated using Cronbach's $\alpha$-test and test-retest reliability using intraclass correlation coefficient (ICC). Data analyses were carried out with SPSS 18.0 for Windows (IBM, Armonk, NY, USA).

\section{Results}

Table 1 shows the demographic and clinical variables of the entire sample of aphasic patients. The 146 aphasic subjects (67 female and 79 male) had a mean age of 68.4 years, a mean education of 6.96 years, and a mean poststroke period before assessment of 399.16 days. Most patients (80.2\%) were married and had a caregiver, 17 aphasic subjects were widowed but had a child as a caregiver, while twelve patients were single. 
Table I Demographic and clinical features of aphasic patients

\begin{tabular}{ll}
\hline Demographic features & \\
Age (years), mean \pm SD & $68.39 \pm 10.29$ \\
Education (years), mean \pm SD & $6.96 \pm 4.27$ \\
Sex (M/F) & $79 / 67$ \\
Marital status, $n(\%)$ & \\
- Married & $117(80.2)$ \\
- Widowed & $17(11.6)$ \\
- Divorced & $5(3.4)$ \\
- Single & $7(4.8)$ \\
Clinical features & \\
Token test & $11.50 \pm 9.36$ \\
Total FIM & $48.52 \pm 28.95$ \\
Motor FIM & $33.76 \pm 23.27$ \\
Cognitive FIM & $14.64 \pm 9.39$ \\
Onset (months), mean \pm SD & $399.16 \pm 605.46$ \\
\hline
\end{tabular}

Abbreviations: SD, standard deviation; FIM, Functional Independence Measure; $\mathrm{M}$, male; $\mathrm{F}$, female.

The language assessment by AAT classified 102 patients as nonfluent PWA (61 with global aphasia, 39 with Broca's aphasia, and two with transcortical motor aphasia), and 38 patients with a fluent form of aphasia ( 25 with Wernicke's aphasia, twelve amnesic, one with a transcortical sensorial aphasia). Six patients had residual aphasic symptoms.

A PCA was conducted on the 37 items with oblique rotation (oblimin). The Kaiser-Meyer-Olkin measure (KMO) verified the sampling adequacy for the analyses $(\mathrm{KMO}=0.924)$, and all $\mathrm{KMO}$ values for individual items were $>0.6$, which is well above the acceptable limit of 0.5 . Bartlett's test of sphericity $-\chi^{2(666)}=4,674.195, P<0.001-$ indicated that correlations between items were sufficiently large for PCA.

The final model explained $59.5 \%$ of the variance and included three factors: the first factor, named communication, comprises 22 items evaluating the patient's ability to express and understand in real life and pragmatic situations; the second factor, named psychological condition, includes six items evaluating the impact of language deficits on emotional status; and the third factor, named autonomy, includes nine items assessing the independence of the subject in activities of daily life. Table 2 reports the factor structure of QLQA.

\section{Reliability}

We assessed the QLQA reliability, ie, the internal consistency and test-retest reliability. To assess the extent to which QLQA items measure the format and homogeneity of the scales, we calculated the internal consistency using Cronbach's $\alpha$-coefficients (Table 3) for the whole scale and for each of the three subscales. The internal consistency of the QLQA was high $(\alpha=0.96$, criteria for acceptability $=$ Cronbach's $\alpha>0.70)$. The QLQA subscales showed similarly high reliability, ranging from 0.79 for psychological condition to 0.97 for communication subscale and 0.89 for autonomy subscale.

The test-retest reliability, defined as the stability of an instrument over time, was evaluated by administering this test at a 3-day interval to a small sample of 14 (10\% of complete sample) aphasic patients with similar characteristics to those of the overall sample in terms of age, sex, marital status, and aphasia. The QLQA was administered to the same caregiver by the different speech therapist. The QLQA showed good test-retest reliability for both overall $(\mathrm{ICC}=0.98$ ) and subscale scores (communication ICC $=0.95$, autonomy ICC $=0.85$, psychological condition ICC $=0.65$ ). ICCs $<0.40$ were seen as indicating poor agreement, $0.40-0.75$ fairto-good agreement, and $0.76-1.00$ excellent agreement. ${ }^{39}$ QLQA total, communication, and autonomy subscales had excellent reliability. The lower test-retest reliability of the psychological condition subscale can be lined up with a physiological variation of the patient's mood state.

\section{Validity \\ Convergent and discriminant validity}

Table 4 reports the correlation coefficients (Spearman's $\rho$ ). The convergent validity was calculated by correlating the QLQA subscales and total scores with tests that assessed the same or similar constructs.

High correlation coefficients were found between QLQA total, the communication and autonomy subscales, and FIM (respectively, $R=0.69, R=0.62, R=0.67$ ) and FAM scores (respectively, $R=0.75, R=0.73, R=0.64$ ). All these scales in fact evaluated the patients' autonomy both for motor and in cognitive aspects. High correlations were found also between QLQA total and communication subscale and AAT scores (respectively, $R=0.58, R=0.63$ ). A significant correlation was found with FAM Emotional Status and Social Interaction subscales and QLQA Psychological Condition subscale.

To evaluate the discriminant validity, we calculated the correlation coefficients between the QLQA total and subscale scores and the scores of tests assessing different functions. Low correlations were found between the QLQA total and subscale scores and Raven's Coloured Progressive Matrices score. For the QLQA psychological condition subscale, we found low correlation coefficients, showing the specificity of this subscale.

\section{Sensitivity to the severity of aphasia}

Subjects with aphasia $(n=146)$ were divided into two groups based on the severity of language disorders, according to 
Table 2 Factor structure of QLQA

\begin{tabular}{|c|c|c|c|}
\hline \multirow[t]{3}{*}{ Items } & \multicolumn{3}{|l|}{ Item loading } \\
\hline & Factor I & Factor 2 & \\
\hline & Communication & Psychological condition & Autonomy \\
\hline To speak with a group & 0.879 & & \\
\hline To speak of a new topic in a conversation & 0.866 & & \\
\hline To follow a conversation with many people & 0.852 & & \\
\hline To take part in a conversation about you & 0.847 & & \\
\hline To tell facts or events & 0.832 & & \\
\hline To understand a joke, proverb, and an expression & 0.821 & & \\
\hline To speak with people not belonging to family & 0.819 & & \\
\hline To express your opinion when you make decisions that concern you & 0.816 & & \\
\hline To understand when others speak & 0.815 & & \\
\hline To explain yourself with your relatives & 0.812 & & \\
\hline To understand television programs & 0.805 & & \\
\hline To understand if others speak quickly & 0.801 & & \\
\hline To discuss a question carefully & 0.788 & & \\
\hline To understand something that does not concern you & 0.779 & & \\
\hline To begin a conversation & 0.763 & & \\
\hline To understand a journal article & 0.748 & & \\
\hline To find the right words & 0.747 & & \\
\hline To use the phone & 0.708 & & \\
\hline To formulate a sentence & 0.657 & & \\
\hline To draw someone's attention in case of need & 0.631 & & \\
\hline To draw someone's attention using gestures and sounds & 0.627 & & \\
\hline To take care of children & 0.542 & & \\
\hline To feel sadness & & 0.769 & \\
\hline The psychological condition affects the social life & & 0.701 & \\
\hline To feel embarrassment when you are with other people & & 0.697 & \\
\hline To feel irritable & & 0.682 & \\
\hline The physical status affects the emotional one & & 0.642 & \\
\hline Language problems affect your work & & 0.512 & \\
\hline To do the shopping & & & -0.858 \\
\hline To go out & & & -0.847 \\
\hline To take pharmacological therapy & & & -0.780 \\
\hline To write & & & -0.723 \\
\hline To manage your money & & & -0.713 \\
\hline Need of someone's assistance in ADL & & & -0.701 \\
\hline To participate in hobbies & & & -0.692 \\
\hline Need of someone's help in daily shopping & & & -0.562 \\
\hline Need of assistance in working & & & -0.457 \\
\hline
\end{tabular}

Abbreviations: QLQA, quality of life questionnaire for aphasics; ADL, activities of daily living.

AAT assessment. A total of 112 patients had severe aphasia, and 34 had mild language disorders. The two groups were comparable for age, education, and time from onset.

We used the Mann-Whitney test to compare the QLQA, linguistic tests, and FIM scores of severe and mild patients. The results are shown in Table 5. Severe patients, apart from having a more severe linguistic deficit, were significantly more impaired in motor and cognitive autonomy, based on FIM data $(P<0.001)$. Significant differences were found in communicative, autonomy, and total QLQA scores $(P<0.001)$ : mild PWA in fact had a better QL than severe patients. The psychological condition score was not different between the two groups.

\section{Sensitivity to time from stroke}

Eighty-four aphasic patients were assessed within 3 months from stroke, and 62 patients were evaluated in a chronic stage of disease (after 3 months). The two groups were different for age $(P<0.01)$ and time from stroke $(P<0.001)$, and were comparable for educational level (Table 6). Linguistic and motor conditions were significantly different between the groups. No difference was found in Raven's test score. QLQA patients evaluated in the acute stage showed greater impairment in communication, autonomy, and total score. The time from stroke helps the aphasic patients to adapt to their new condition. No difference between the two groups was found in psychological condition. 
Table 3 Convergent and discriminant validity of QLQA

\begin{tabular}{|c|c|c|c|c|}
\hline Instruments & $\begin{array}{l}\text { QLQA } \\
\text { communication }\end{array}$ & $\begin{array}{l}\text { QLQA } \\
\text { autonomy }\end{array}$ & $\begin{array}{l}\text { QLQA psychological } \\
\text { condition }\end{array}$ & $\begin{array}{l}\text { QLQA } \\
\text { total score }\end{array}$ \\
\hline Token test & $0.60 * *$ & $0.35 * *$ & $0.23 * *$ & $0.59 * *$ \\
\hline Raven's coloured progressive matrices & $0.28^{*}$ & 0.22 & -0.00 & 0.25 \\
\hline Total FIM & $0.62 * *$ & $0.67^{* *}$ & 0.13 & $0.69 * *$ \\
\hline Motor FIM & $0.52 * *$ & $0.63 * *$ & 0.11 & $0.63 * *$ \\
\hline Cognitive FIM & $0.65 * *$ & $0.53^{* *}$ & 0.14 & $0.64 * *$ \\
\hline FAM comprehension & $0.63 * *$ & $0.42^{* *}$ & 0.11 & $0.63^{* *}$ \\
\hline FAM expression & $0.44 * *$ & $0.40 * *$ & 0.09 & $0.37 * *$ \\
\hline FAM reading & $0.63 * *$ & $0.58^{* *}$ & $0.20^{*}$ & $0.59 * *$ \\
\hline FAM writing & $0.54 * *$ & $0.6 I^{* *}$ & $0.18^{*}$ & $0.60 * *$ \\
\hline FAM speech intelligibility & $0.55 * *$ & $0.48 * *$ & $0.21 *$ & $0.54 * *$ \\
\hline FAM social interaction & $0.62 * *$ & $0.5 I^{* *}$ & $0.17^{*}$ & $0.62 * *$ \\
\hline FAM emotional status & $0.58 * *$ & $0.43 * *$ & $0.29 * *$ & $0.60 * *$ \\
\hline FAM adjustment to limitation & $0.61^{* *}$ & $0.49 * *$ & 0.04 & $0.7 I^{* *}$ \\
\hline FAM employability & $0.50 * *$ & $0.63 * *$ & $0.22 * *$ & $0.5 I^{* *}$ \\
\hline FAM problem solving & $0.44 * *$ & $0.53^{* *}$ & 0.16 & $0.36 * *$ \\
\hline FAM memory & $0.60 * *$ & $0.53 * *$ & 0.07 & $0.63 * *$ \\
\hline FAM orientation & $0.65 * *$ & $0.54 * *$ & 0.12 & $0.69 * *$ \\
\hline FAM attention span & $0.54 * *$ & $0.57^{* *}$ & $0.17^{*}$ & $0.57^{* *}$ \\
\hline FAM safety judgment & $0.54 * *$ & $0.5 I^{* *}$ & 0.03 & $0.61 * *$ \\
\hline FAM total score & $0.73 * *$ & $0.64 * *$ & $0.17^{*}$ & $0.75 * *$ \\
\hline AAT spontaneous speech total score & $0.63 * *$ & $0.42^{* *}$ & 0.15 & $0.58 * *$ \\
\hline
\end{tabular}

Notes: $* P<0.05 ; * * P<0.001$.

Abbreviations: QLQA, quality of life questionnaire for aphasics; FIM, Functional Independence Measure; FAM, Functional Assessment Measure; AAT, Aachener Aphasia Test.

\section{Differences between PWA and control subjects}

Finally, we evaluated the specific role of linguistic deficits on QL in two groups of patients with and without aphasia in the acute stage of disease. Since the time from stroke has an influence on QL of patients, we chose to compare the QLQA scores of 84 acute PWA with hemiplegia to those of a control group of 37 subjects with motor impairment and without aphasic problems, assessed within 3 months from stroke. The demographic and clinical variables are described

Table 4 Demographic and clinical features of severe and mild aphasics

\begin{tabular}{|c|c|c|c|}
\hline & $\begin{array}{l}\text { Severe aphasics } \\
(n=\mid 12)\end{array}$ & $\begin{array}{l}\text { Mild aphasics } \\
(n=34)\end{array}$ & $\begin{array}{l}\text { Comparison between } \\
\text { two groups }\end{array}$ \\
\hline \multicolumn{4}{|l|}{ Demographic features } \\
\hline Age (years), mean \pm SD & $68.4 I \pm 9.83$ & $68.32 \pm 11.88$ & $t_{144}=-0.043, \mathrm{NS}$ \\
\hline Education (years), mean \pm SD & $6.79 \pm 4.35$ & $7.15 \pm 4.21$ & $t_{144}=0.428, \mathrm{NS}$ \\
\hline $\operatorname{Sex}(M / F)$ & $61 / 51$ & $18 / 16$ & $\chi^{2}=0.02, d f=I, N S$ \\
\hline \multicolumn{4}{|l|}{ Clinical features, mean \pm SD } \\
\hline Onset, months & $384.48 \pm 629.03$ & $447.09 \pm 526.92$ & $t_{143}=0.526, \mathrm{NS}$ \\
\hline Token test & $8.80 \pm 7.62$ & $19.13 \pm 9.72$ & $U=669 P<0.001$ \\
\hline Raven's coloured matrices & $16.57 \pm 6.54$ & $21.94 \pm 8.55$ & $U=|89.5, P<0.0|$ \\
\hline Total FIM & $41.66 \pm 24.51$ & $70.32 \pm 31.43$ & $U=82 \mathrm{I}, P<0.00 \mathrm{I}$ \\
\hline Motor FIM & $29.66 \pm 20.88$ & $46.79 \pm 25.88$ & $U=I, 079.5, P<0.00 \mathrm{I}$ \\
\hline Cognitive FIM & $11.83 \pm 6.29$ & $23.56 \pm 11.87$ & $U=517, P<0.00 \mathrm{I}$ \\
\hline QLQA communication & $29.46 \pm 19.67$ & $53.55 \pm 20.27$ & $U=772.5, P<0.00 \mathrm{I}$ \\
\hline QLQA autonomy & $4.39 \pm 5.49$ & $12.94 \pm 9.86$ & $U=832.5, P<0.00 \mathrm{I}$ \\
\hline QLQA psychological condition & $7.34 \pm 5$ & $9.12 \pm 6.31$ & $U=I, 588.5, \mathrm{NS}$ \\
\hline QLQA total score & $46.75 \pm 27.32$ & $77.5 \pm 28.59$ & $U=858, P<0.001$ \\
\hline AAT total score & $7.65 \pm 8.31$ & $19.65 \pm 3.99$ & $U=188.5, P<0.001$ \\
\hline
\end{tabular}

Abbreviations: QLQA, quality of life questionnaire for aphasics; SD, standard deviation; NS, not significant; $d f$, degrees of freedom; FIM, Functional Independence Measure; AAT, Aachener Aphasia Test; M, male; F, female; NS, not significant. 
Table 5 Demographic and clinical features of acute and chronic aphasics

\begin{tabular}{|c|c|c|c|}
\hline & $\begin{array}{l}\text { Acute aphasics } \\
(n=84)\end{array}$ & $\begin{array}{l}\text { Chronic aphasics } \\
(n=62)\end{array}$ & $\begin{array}{l}\text { Comparison between } \\
\text { two groups }\end{array}$ \\
\hline \multicolumn{4}{|l|}{ Demographic features } \\
\hline Age (years), mean \pm SD & $70.46 \pm 9.98$ & $65.58 \pm 10.12$ & $t_{144}=2.904, P<0.01$ \\
\hline Education (years), mean \pm SD & $6.67 \pm 4.49$ & $7.15 \pm 4.05$ & $t_{144}=-0.663, \mathrm{NS}$ \\
\hline $\operatorname{Sex}(M / F)$ & $41 / 43$ & $38 / 24$ & $\chi^{2}=1.76, d f=I, N S$ \\
\hline \multicolumn{4}{|l|}{ Clinical features, mean \pm SD } \\
\hline Onset, months & $30.31 \pm 16.55$ & $907.08 \pm 653.20$ & $t_{143}=-12.313, P<0.001$ \\
\hline Token test & $9.27 \pm 9.23$ & $14.03 \pm 8.93$ & $U=I, 435.5, P<0.0 \mathrm{I}$ \\
\hline Raven's coloured matrices & $18.16 \pm 7.64$ & $20.12 \pm 7.94$ & $U=95, N S$ \\
\hline Total FIM & $36.33 \pm 21.59$ & $66.17 \pm 29.21$ & $U=927.5, P<0.001$ \\
\hline Motor FIM & $23.82 \pm 16.89$ & $48.16 \pm 23.83$ & $U=854, P<0.00 \mathrm{I}$ \\
\hline Cognitive FIM & $|2.3| \pm 7.49$ & $18.02 \pm 10.82$ & $U=I, 479, P<0.00 I$ \\
\hline QLQA communication & $27.01 \pm 20.02$ & $46.00 \pm 20.46$ & $U=|, 3| 6.5, P<0.00 \mid$ \\
\hline QLQA autonomy & $4.08 \pm 5.03$ & $9.5 \pm 9.34$ & $U=I, 582.5, P<0.00 \mathrm{I}$ \\
\hline QLQA psychological condition & $7.55 \pm 5.44$ & $8.03 \pm 5.29$ & $U=2,43 \mathrm{I} .5, \mathrm{NS}$ \\
\hline QLQA total score & $38.64 \pm 23.79$ & $74.6 \pm 26.06$ & $U=803.5 P<0.00 \mathrm{I}$ \\
\hline AAT total score & $10.85 \pm 9.06$ & $6.82 \pm 8.65$ & $U=3 \mid 4, \mathrm{NS}$ \\
\hline
\end{tabular}

Abbreviations: SD, standard deviation; NS, not significant; $d f$, degrees of freedom; FIM, Functional Independence Measure; QLQA, quality of life questionnaire for aphasics; AAT, Aachener Aphasia Test; M, male; F, female; NS, not significant.

in Table 6. The two groups were compared in the following series of tests:

- QLQA

- Token Test

- Raven's Coloured Progressive Matrices

- FIM. ${ }^{17,20}$

The control patients had a mean age of 64.79 years, a mean education of 7.69 years, and a mean time from stroke of 114.19 days.

PWA and controls were similar for age, educational level, sex, and motor autonomy. Significant differences were found in time from stroke $(P<0.001)$, cognitive $(P<0.001)$ and total FIM scores $(P<0.001)$, and Token Test $(P<0.001)$. No difference was found in Raven's matrices score and FIM motor score. Statistically significant differences between PWA and control subjects were found regarding QLQA total and subscale scores (communication, autonomy, and psychological condition).

\section{Discussion}

The results of this preliminary psychometric examination suggest that the QLQA is a reliable and valid measure of

Table 6 Demographic and clinical variables of aphasics and control group

\begin{tabular}{|c|c|c|c|}
\hline & $\begin{array}{l}\text { Aphasic patients } \\
(n=84)\end{array}$ & $\begin{array}{l}\text { Control patients } \\
(n=37)\end{array}$ & $\begin{array}{l}\text { Comparison between } \\
\text { two groups }\end{array}$ \\
\hline \multicolumn{4}{|l|}{ Demographic features } \\
\hline Age (years), mean $\pm S D$ & $70.46 \pm 9.99$ & $67.24 \pm 7.70$ & $t_{119}=1.745, \mathrm{NS}$ \\
\hline Education (years), mean $\pm S D$ & $6.67 \pm 4.49$ & $7.22 \pm 4.76$ & $t_{119}=-0.609, \mathrm{NS}$ \\
\hline $\operatorname{Sex}(M / F)$ & $4 I / 43$ & $22 / 15$ & $\chi^{2}=0.78, d f=I, N S$ \\
\hline \multicolumn{4}{|l|}{ Clinical features, mean \pm SD } \\
\hline Onset, months & $30.31 \pm 16.56$ & $43.05 \pm 20.43$ & $t_{119}=-3.624, P<0.001$ \\
\hline Token test & $9.27 \pm 9.23$ & $28.88 \pm 4.01$ & $U=93.5, P<0.001$ \\
\hline Raven's coloured matrices & $18.16 \pm 7.64$ & $20.28 \pm 5.14$ & $U=673, \mathrm{NS}$ \\
\hline Total FIM & $36.33 \pm 21.59$ & $48.08 \pm 16.02$ & $U=809, P<0.001$ \\
\hline Motor FIM & $23.82 \pm 16.88$ & $24.19 \pm 12.2$ & $U=1243.5, \mathrm{NS}$ \\
\hline Cognitive FIM & $|2.3| \pm 7.49$ & $24.16 \pm 7.74$ & $U=433.5, P<0.00 \mathrm{I}$ \\
\hline QLQA communication & $27.01 \pm 20.02$ & $71.67 \pm 18.10$ & $U=206, P<0.00 \mathrm{I}$ \\
\hline QLQA autonomy & $4.08 \pm 5.03$ & $16.86 \pm 9.06$ & $U=309, P<0.001$ \\
\hline QLQA psychological condition & $7.55 \pm 5.44$ & $18.94 \pm 5.4$ & $U=268, P<0.00 \mathrm{I}$ \\
\hline QLQA total score & $38.64 \pm 23.79$ & $107.49 \pm 28.53$ & $U=|46, P<0.00|$ \\
\hline
\end{tabular}

Abbreviations: SD, standard deviation; NS, not significant; $d f$, degrees of freedom; FIM, Functional Independence Measure; QLQA, quality of life questionnaire for aphasics; $M$, male; $F$, female. 
QL in people with aphasia. The internal consistency was found to be high. The data of convergent and discriminant validity are not univocal: the correlation coefficients are not very high, and sometimes there were correlations also with different measures. This could be due to the ecological value of the QLQA: as in daily life, QLQA comprehension is influenced and improved by pragmatic aids, unlike Token Test performances, causing the absence of any relationship between PWA subjective estimation of their comprehension difficulties and the Token Test results. ${ }^{41}$ Moreover, also other measures used for this study, such as FAM, evaluate the patient's functional autonomy in multiple cognitive domains that do not leave out of consideration the use of language. This could explain, for example, the correlations that we found between QLQA total, subscales, and FAM subscales.

The QLQA differs from other QL questionnaires due to the greater importance given to the reduction of autonomy caused by aphasia, independently of motor deficits, as shown by the differences between PWA and controls. This aspect differentiates our questionnaire from the other QL measures, such as SAQOL-39. This scale considers the physical autonomy of PWA in daily living activities.

Our results showed that the time from stroke and the severity of aphasia influence the patient's functional and communicative autonomy, but not the psychological condition. The mild and the chronic aphasic patients had a better quality of life than severe and acute ones, underlining the fact the passing of time helps patients with language disorders to adapt themselves to the new condition. In the literature, other studies, ${ }^{20,42}$ reported low-to-moderate improvements in QL after stroke. The physical and communication domains present greater improvement between acute stroke and 3 months, while psychosocial well-being takes longer to improve poststroke. Regarding severity of language disease, Engell et $\mathrm{al}^{35}$ found a correlation between the ALQI ratings and the performance scores of the AAT. Total, physical, and psychosocial scores were significantly correlated with communicative and systematic failures in spontaneous language, but not with articulation disorders.

Moreover, the aphasia has an important role in the emotional status of patients. Subjects with aphasia, independently of severity and time, tend towards social isolation and demand that other family members manage personal and family problems. Our results agree with many studies in the literature that underline that the frequency of depression in aphasic patients is higher than in other stroke survivors. ${ }^{9,43}$

This test was given to PWA in the presence of a caregiver and with the aid of a speech therapist to overcome comprehension/expression deficits and avoid the problem of missing data. This could be a valid method when using these questionnaires in future, since severe PWA have until now been excluded from similar evaluations. Other studies ${ }^{18}$ suggested that proxy respondents can provide reliable information on the HRQL of PWA at the chronic stage of disease, even if self-report is more valid than any proxy report. However, using proxies may be a useful way to obtain information on the HRQL of patients with severe aphasia.

With QLQA, the differences among emotional, social, and communicative aspects of PWA and patients with motor deficits clearly emerge. This has an important effect on rehabilitation: on the one hand, it justifies the equal importance of language and motor rehabilitation; on the other, it explains the reasons for a greater incidence of stress among PWA and their family members. ${ }^{44} \mathrm{HRQL}$ scales are essential in stroke assessment and outcome measurement. With patients' subjective evaluation of their functioning and well-being, the speech-language pathologists and related professionals get a more holistic picture of how stroke and aphasia has affected patients' lives, and they can make more informed decisions on what needs to be targeted in intervention. Lastly, these findings suggest that people with stroke, and particularly those with aphasia, need long-term service provision that takes into account their affected mood, through community-based interventions, eg, participation in personally relevant meaningful activities.

The use of QLQA in the assessment of people with aphasia focuses on the frequency of activities of life, depending on the correct use of language. It may point out gains in linguistic and communicative behaviors, due to rehabilitation training and/or personal ability of coping with disease limitation, and it shows daily personal aspects of discomfort, which are rarely evidenced by the patients, while leading them to isolation and passivity. Timely interventions by speech-language pathologists and occupational therapists, psychological support to patients and caregivers, and changes in communicative environments are needed to promote socialization, according to the conceptual models of the ICF. As aphasia causes communication impairment and altered relationships, for aphasic people the aim of rehabilitation should be to improve either the disability (making language and communication more efficient) or the possibilities of coping behaviors, intervening in the social sphere, and reinforcing self-esteem and the desire to return to personal autonomy.

A limitation of this study lies in the comparison of the QLQA with measures of functional autonomy. It could be interesting also to compare the QLQA psychological 
condition subscale with scales of mood state, in order to increase the construct validity of our questionnaire.

As is common with new measures, further research is needed to confirm its psychometric properties in independent samples. Moreover, it is necessary to evaluate the test-retest reliability in a larger sample of PWA. Our next objective is to evaluate the QL of aphasic patients through a multicentric longitudinal study, in order to assess how QL changes with the evolution of language disease.

\section{Conclusion}

QLQA is a valid measure of QL in PWA, contributing to a better distinction between severe and mild aphasia and also to the variations in QL depending on the time interval from stroke. Improvement in the severity of language deficits also causes an improvement in QL, except for the psychological condition. This underlines the important role of aphasia in social isolation and emotional distress of patients.

The regular use of QL scales in the assessment of PWA highlights their disability, handicap, and ability to cope. QLQA would be a useful tool in planning rehabilitation with a view to achieving greater functional autonomy in social and communicative environments.

\section{Acknowledgments}

We are grateful to Fara Cellamare and Elisabetta Cafforio, speech and language therapists, who were involved in recruitment and in proposing the questionnaire. Thank you to the Italian Aphasia Association (AITA, Puglia, Italy) members and their families who agreed to participate in this research project.

\section{Author contributions}

All authors participated in the design of the study and the analysis. This article has been read and revised by all authors. All authors read and approved the final manuscript.

\section{Disclosure}

The authors report no conflicts of interest in this work.

\section{References}

1. Hilari K, Needle JJ, Harrison KL. What are the important factors in health-related quality of life for people with aphasia? A systematic review. Arch Phys Med Rehabil. 2012;93:S86-S95.

2. Ones K, Yilmaz E, Cetinkaya B, Caglar N. Quality of life for patients post-stroke and the factors affecting it. J Stroke Cerebrovasc Dis. 2005;6:261-266.

3. Rønning OM, Stovem K. Determinants of change in quality of life from 1 to 6 months following acute stroke. Cerebrovasc Dis. 2008;25: $67-73$.
4. Taylor-Sarno M. Preliminary findings in a study of age, linguistic evolution and quality of life in recovery from aphasia. Scand J Rehab Med Suppl. 1992;26:43-59.

5. Bays CL. Quality of life of stroke survivors: a research synthesis. J Neurosci Nurs. 2001;33:310-316.

6. Wyller TB, Sveen U, Sadring KM, Pettersen AM, Bautz-Holter E. Subjective well-being one year after stroke. Clin Rehabil. 1997;11: 139-145.

7. King RB. Quality of life after stroke. Stroke. 1996;27:1467-1472.

8. Hemsley $\mathrm{G}$, Code $\mathrm{C}$. Interactions between recovery in aphasia, emotional and psychosocial factors in subjects with aphasia, their significant others and speech pathologists. Disabil Rehabil. 1996;18:567-584.

9. Kauhanen ML, Korpelainen JT, Hiltunen P, et al. Aphasia, depression, and non-verbal cognitive impairment in ischaemic stroke. Cerebrovasc Dis. 2000;10:455-461.

10. Parr S. Living with severe aphasia: tracking social exclusion. Aphasiology. 2007;21:98-123.

11. Cruice M, Worrall L, Hickson L. Quantifying aphasic people's social lives in the context of non-aphasic peers. Aphasiology. 2006;20: $1210-1225$.

12. Hilari K, Northcott S. Social support in people with chronic aphasia. Aphasiology. 2006;20:17-36.

13. Hilari K, Wiggins RD, Roy P, Byng S, Smith SC. Predictors of health-related quality of life (HRQL) in people with chronic aphasia. Aphasiology. 2003;17:365-381

14. Ross KB, Wertz RT. Quality of life with and without aphasia. Aphasiology. 2003;34:355-364.

15. Franzén-Dahlin A, Rydell Karlsson M, Mejhert M, Laska AC. Quality of life in chronic disease: a comparison between patients with heart failure and patients with aphasia after stroke. J Clin Nurs. 2010;19:1855-1860.

16. Lam JM, Wodchis WP. The relationship of 60 disease diagnoses and 15 conditions to preference-based health-related quality of life in Ontario hospital-based long-term care residents. Med Care. 2010;48: 380-387.

17. Le Dorze G, Brassard C. A description of the consequences of aphasia on aphasic persons and their relatives and friends, based on the WHO model of chronic diseases. Aphasiology. 1995;9:239-255.

18. Hilari K, Owen S, Farrelly SJ. Proxy and self-report agreement on the Stroke and Aphasia Quality of Life Scale-39. J Neurol Neurosurg Psychiatry. 2007;78:1072-1075.

19. Cruice M, Worrall L, Hickson L, Murison R. Measuring quality of life: Comparing family members' and friends' ratings with those of their aphasic partners. Aphasiology 2005;19:111-129.

20. Hilari K, Lamping DL, Smith SC, Northcott S, Lamb A, Marshall J. Psychometric properties of the Stroke and Aphasia Quality of Life Scale (SAQOL-39) in a generic stroke population. Clin Rehabil. 2009;23: 544-557.

21. Luzzatti C, Willmes K, De Blesere R. L'Aachener Aphasie Test (AAT), Versione Italiana. Manuale e Dati Normativi. 2nd ed. Florence: Organizzazione Speciali; 1996.

22. DeRenzi E, Vignolo LA. The token test: a sensitive test to detect receptive disturbances in aphasics. Brain. 1962;85:665-678.

23. Raven JC, Court J, Raven J. Raven Manual: Colored Progressive Matrices. London: Oxford Psychologists Press; 1995.

24. Basso A, Capitani E, Laiacona M. Raven's coloured progressive matrices: normative values on 305 adult controls. Funct Neurol. 1987;2:189-194.

25. Linacee JW, Heinemann AW, Wright BD, Granger C, Hamilton BB. The structure and stability of the functional independence measure. Arch Phys Med Rehabil. 1994;75:127-132.

26. [No authors listed]. FIM versione italiana, manuale d'uso. Ricerca in Riabilitazione. 1993;2:1-44.

27. Hall KM. Functional Assessment Measure. General Rehabilitation and Traumatic Brain Injury Program Evaluation. 1992; San Jose, CA, USA: Santa Clara Valley Medical Center. 
28. Hall KM, Hamilton BB, Gordon WA, Zasler ND. Characteristics and comparisons of functional assessment indices: Disability Rating Scale, Functional Independence Measure and Functional Assessment Measure. J Head Trauma Rehabil. 1993;8:60-74.

29. Tesio L, Cantagallo A. The functional assessment measure (FAM) in closed traumatic brain injury outpatients: a Rasch-based psychometric study. J Outcome Meas. 1998;2:79-96.

30. World Health Organization. The International Classification of Functioning, Disability and Health. Geneva: WHO; 2001.

31. Glueckauf RL, Blonder LX, Ecklund-Johnson E, Maher L, Crosson B, Gonzalez-Rothi L. Functional Outcome Questionnaire for Aphasia: overview and preliminary psychometric evaluation. Neuro Rehabilitation. 2003;18:281-290.

32. Hilari K, Byng S, Camping DL, Smith SC. Stroke and Aphasia Quality of Life Scale-39 (SAQOL-39): evaluation of acceptability, reliability, and validity. Stroke. 2003;34:1944-1950.

33. Posteraro L, Formis A, Bidini C, et al. Aphasia quality of life: reliability of the Italian version of SAQOL-39. Eura Medicophys. 2004;4: 257-262.

34. Lata-Caneda MC, Piñeiro-Temprano M, García-Fraga I, García-Armesto I, Barrueco-Egido JR, Meijide-Failde R. Spanish adaptation of the Stroke and Aphasia Quality of Life Scale-39 (SAQOL-39). Eur J Phys Rehabil Med. 2009;45:379-384.

35. Engell B, Hütter BO, Willmes K, Huber W. Quality of life in aphasia: validation of a pictorial self-rating procedure. Aphasiology. 2003;17: 383-396.
36. Bergner M, Bobitt RA, Carter WB, Gilson BS. The Sickness Impact Profile: development and final revision of a health status measure. Med Care. 1981;19:787-805.

37. Hütter BO. Sickness Impact Profile (SIP)-German version. In: Salek S, editor. Compendium of Quality of Life Instruments. Chichester, UK: John Wiley \& Sons; 2001.

38. Streiner DL, Norman GR. Health Measurement Scales: A Practical Guide to Their Development and Use. New York: Oxford University Press; 1995.

39. Aaronson N, Alonso J, Burnam A, et al. Assessing health status and quality of life instruments: attributes and review criteria. Qual Life Res. 2002;11:193-205.

40. Rosner B. Fundamentals of Biostatistics. 6th Ed. Pacific Grove (CA): Duxbury; 2000.

41. Caporali A, Basso A. A survey of long-term outcome of aphasia and of changes of gainful employment. Aphasiology. 2003;17:815-834.

42. Samsa GP, Matchar DB. How strong is the relationship functional status and quality of life among persons with stroke? J Rehabil Res Dev. 2004;41:279-282.

43. Hackett ML, Yapa C, Parag V, Anderson CS. Frequency of depression after stroke. A systematic review of observational studies. Stroke. 2005;36:1330-1340

44. Bakas T, Kroenke K, Plue L, Perkins S, Williams L. Outcomes among family caregivers of aphasics versus nonaphasic stroke survivors. Rehabil Nurs. 2006;31:33-42.
Neuropsychiatric Disease and Treatment

\section{Publish your work in this journal}

Neuropsychiatric Disease and Treatment is an international, peerreviewed journal of clinical therapeutics and pharmacology focusing on concise rapid reporting of clinical or pre-clinical studies on a range of neuropsychiatric and neurological disorders. This journa is indexed on PubMed Central, the 'PsycINFO' database and CAS.

\section{Dovepress}

The manuscript management system is completely online and includes a very quick and fair peer-review system, which is all easy to use. Visit http://www.dovepress.com/testimonials.php to read real quotes from published authors. 